DOI 10.1007/s10039-017-0287-1

Online publiziert: 25 . Juli 2017

(c) Springer Medizin Verlag GmbH 2017

CrossMark

\title{
Notwendige Weiterentwicklung aus der Sicht des D-Arztes am Krankenhaus
}

Das stationäre Heilverfahren wurde mit Januar 2014 neu ausgerichtet. Ziele der Neuordnung im stationären Bereich waren eine Konzentration der Versorgung von Schwerverletzten auf qualifizierte und erfahrene Kliniken, eine Differenzierung der Heilverfahren nach Art und Schwere der Verletzungsart, eine Profilierung von Qualitätsanforderungen und eine Stärkung der sektorenübergreifenden Versorgung im Sinne der Unfallversicherungsträger. Dazu wurden sowohl bauliche wie fachliche Strukturanforderungen an die Kliniken gestellt, damit das Behandlungsniveau gesteigert werden konnte. Erste Ergebnisse zeigen, dass es durch die Veränderung in den BG (Berufsgenossenschaft)-Kliniken $\mathrm{zu}$ einer Zunahme der SAV (Schwerverletztenartenverfahren)-Patienten gekommen ist $[2,4]$.

Vorbild für die Neuausrichtung war das Weißbuch Schwerverletztenversorgung der DGU (Deutsche Gesellschaft für Unfallchirurgie) aus dem Jahr 2006. Durch die dreistufigen Traumazentren sollte die Versorgung Schwerverletzter verbessert werden. Schon nach kurzer Zeit konnten dadurch z. B. Verbesserungen der Struktur- und Prozessqualität in den regionalen Netzwerken nachgewiesen werden. Dazu zählen vereinheitlichte Schockraumausstattungen, Implementierung von interdisziplinären $\mathrm{Be}$ handlungsleitlinien und eine verbesserte Kooperation zwischen den Zentren [12]. Bei Teilnahme als Traumazentrum waren die Kliniken verpflichtet, die Behandlungsdaten von Unfallverletzten in das TraumaRegister DGU ${ }^{\circledR}$ einzupflegen.

Bei dem neuen Heilverfahren wurde in Anlehnung an die Traumazentren auch ein Dreistufenmodell etabliert. Neben dem Durchgangsarzt am Krankenhaus (DAV) und dem Verletzungsartenverfahren (VAV) wurde ein SAV eingeführt. Bei den Anforderungen an eine SAV-Klinik orientierte man sich an Vorgaben aus dem Weißbuch für überregionale Traumazentren. Notwendig war es daher, das bisherige Verletzungsartenverzeichnis zu überarbeiten und zu differenzieren. Im Gegensatz zu dem Weißbuch bestand ab Neuausrichtung des Heilverfahrens den Unfallversicherungsträgern die Möglichkeit die Behandlung des Patienten zu überwachen und zu steuern. Erste Publikationen zeigen, dass es zu Veränderungen der Patientenströme gekommen ist mit Verlagerung von SAV-Patienten an Universitätskliniken und überregionale Traumazentren [2-4].

Veränderungen zur Verbesserung der Patientenbehandlung sind immer notwendig und wünschenswert. Genauso ist es aber notwendig, dass die dafür erforderlichen Ressourcen mit verändert werden. Dies findet zum Teil statt, aber es gibt etliche Bereiche, wo notwendige Weiterentwicklungen bisher nicht stattgefunden haben. Dies betrifft unter anderem

- die Bürokratie,

- die Qualität,

- die Vergütungsstruktur und

- die Mitbeteiligung bei der Ausbildung.

\section{Bürokratie}

Mit der Einführung eines elektronischen Datenübermittlungsverfahrens (DALEUV) Anfang dieses Jahrhunderts war eines der Ziele, die Bürokratie zu ver- meiden und die Effizienz zu erhöhen ([1, 7]; - Abb. 1). Schon kurz nach Einführung des Verfahrens berichtete Kleinschmidt [10] über deutliche Kommunikationsschwierigkeiten zwischen der BG und niedergelassenen Ärzten. Es haperte an der Weiterleitung der Berichte an die verschiedenen Berufsgenossenschaften oder Hausärzte, es bestanden personelle Unzulänglichkeiten in der Verwaltung, und D-Berichte mussten nachträglich angefordert werden. Es ist zu vermuten, dass sich dies in den letzten Jahren verbessert hat. Daten dazu liegen aber nicht vor. Aus eigener Erfahrung und im Gespräch mit Kollegen bestehen aber immer noch viele Schwierigkeiten bei dem System. Dies fängt schon bei der Datenübertragung an. Immer wieder kommt es zu Problemen mit der Software, weshalb eine schnelle Datenübermittlung nicht möglich ist. Daraus resultiert, dass möglicherweise z. B. der weiterbehandelnde Kollege keinen Bericht bei Vorstellung des Patienten vorliegen hat. Dies ist aber auch vice versa der Fall. Aber auch ohne Softwareprobleme ist die Datenübermittlung an die weiterbehandelnden Kollegen suboptimal. Es besteht der Eindruck, dass DALE-UV eine Einbahnstraße ist. Um Verbesserungen herbeizuführen, sollte zunächst eine Analyse vorgenommen werden, ob diese oder andere Schwierigkeiten mit dem System häufig und bei einer Vielzahl auftreten. Wenn sich dies bestätigt, müssen intelligente und einfache Lösungen zur besseren und schnelleren Datenübermittlung erarbeitet werden. In der IT-Branche gibt es sicherlich eine Menge an Möglichkeiten, wo kreative Lösungen gemeinsam erarbeitet 


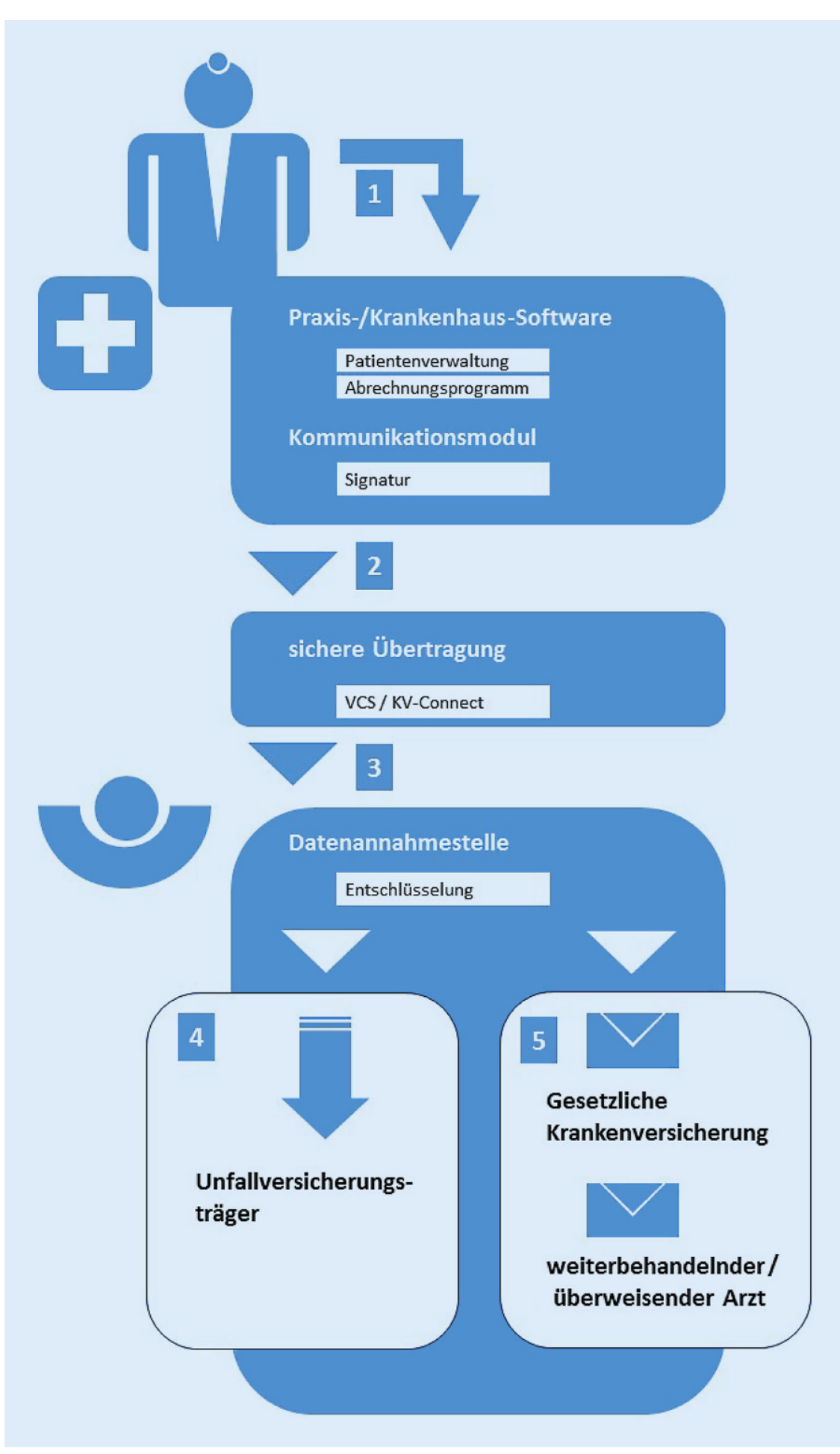

Abb. 1 \ DALE-UV (elektronisches Datenübermittlungsverfahren)-Übertragungsweg. KV Krankenversicherung

werden können. Mit modernen Sprachsteuerungssystemen, wie z. B. SIRI oder ALEXA, könnten möglicherweise Datenübermittlungen vereinfacht werden. Sicherlich gibt es auch eine Vielzahl von Einwänden an Sprachsteuerungssyste- des Systems kommen. Diese Schwierigkeit besteht z. B. bei der Teleradiologie in den Traumanetzwerken. Hier gibt es kein einheitliches System. Sicherlich hat dies auch mit den anfallenden Kosten zu tun. Die Anschaffungskosten der Software müssen meistens von den Kliniken getragen werden, ohne dass dadurch ein Mehrnutzen gegeben ist. Bedingung für die Etablierung eines neuen Systems ist also, dass dieses für die Anwender kostenfrei ist. Ein weiteres notwendiges Entwicklungsfeld ist die Automatisierung. Bei der Anwendung von DALE-UV müssen heutzutage die ICD-Nummern eingegeben werden. Ein automatisiertes Programm, das nach Eingabe der Diagnose die ICD-Nummer automatisch anzeigt, würde zu einer Reduktion der Arbeitsbelastung führen.

Die Dokumentation war und ist eine der Hauptaufgaben der ärztlichen Mitarbeiter jeder Klinik. Wenige Kliniken werden eine Dokumentationskraft zur Verfügung haben, die bürokratische Tätigkeiten erledigt. Durch den zunehmenden ökonomischen Druck in den Krankenhäusern ist es in den letzten Jahren auch $\mathrm{zu}$ einer Veränderung der personellen Situation gekommen mit häufigem Stellenabbau. Der Dokumentationsaufwand hat aber im Gegensatz in vielen Bereichen zugenommen. Dies hat dazu geführt, dass die Assistenten mit der Arbeit unzufriedener geworden sind. Eine aktuelle Umfrage des Hartmannbundes zeigt, dass von 1330 befragten Assistenzärzten fast $37 \%$ mehr als $3 \mathrm{~h}$ täglich Zeit für die Dokumentation aufbringen [8].

Ein zukünftiges Ziel der Unfallversicherungsträger sollte es sein, den Bürokratieabbau für das ärztliche Personal voranzubringen. In anderen medizinischen Bereichen ist dies im Aufbau. Das Ministerium für Gesundheit möchte die Pflegedokumentation entbürokratisieren [5]. Wünschenswert wäre es, wenn auch hier die Unfallversicherungsträger gemeinsam mit den Ärzten Lösungen entwickeln, damit nicht nur der bürokratische Aufwand reduziert wird, sondern auch die Zufriedenheit der Ärzte wieder wächst. 


\section{Qualität}

Mit der Neuausrichtung der Heilverfahren sollten Voraussetzungen geschaffen werden, neue Wege in der Qualitätssicherung zu beschreiten. Die erstklassige Qualität medizinischer Behandlung ist ein wichtiges Ziel. Eine Qualitätstransparenz wird in Anbetracht bestehender Defizite im Krankenhaus immer wichtiger. Das Ergebnis der Wahl ist jedoch nur so gut, wie die Kriterien tauglich sind, gute von schlechter Qualität zu trennen. Quantitative Angaben ohne Vergleichsgrößen sind schwer zu interpretieren. Hohe Fallzahlen gelten zwar als ein Hinweis auf hohe Qualität, es ist aber nicht klar, welche Fallzahlen eine ordentliche Qualität erwarten lassen, was als $\mathrm{zu}$ selten gelten könnte und ob es evtl. auch eine kritische Marke für zu viele Operationen geben könnte. Das Gleiche gilt für die Fallzahlen bei den Untersuchungs- und Behandlungsmethoden. Mit dem Dreistufenmodell wurden Kriterien festgelegt, um die Behandlungsqualität der Patienten zu verbessern. Bisher liegen keine Daten vor, dass es durch diese Veränderung zu einer Verbesserung der Qualität gekommen ist. Es gab auch schon vor Einführung des Heilverfahrens keine Status-quo-Erhebung über die Behandlungsqualität. Es stellt sich die Frage: Wie kann man denn die Qualität überhaupt messen? Nach den derzeitigen vorliegenden Publikationen hat man den Eindruck, dass dies allein durch Zentrierung der Patienten in SAV-Häuser und Universitätskliniken geschieht. Immer wieder liegen Einzelfallberichte vor mit „schlechter" Versorgung in einem kleineren Krankenhaus. Sicherlich müssen zur optimalen Behandlung in großen Kliniken viel mehr Ressourcen vorgehalten werden. Es besteht aber die Gefahr, dass es im Laufe der Zeit auch zu einer Verschiebung anderer Patienten, die keinen Berufsunfall hatten, kommen wird. Wie Sarkar [13] in seiner Publikation schreibt, haben auch gesetzlich krankenversicherte Patienten den gleichen Anspruch auf eine qualitativ hochwertige Versorgung wie der berufsgenossenschaftliche Versicherte. Es wird sicherlich nicht mehr lange dauern, bis auch die gesetzlichen Kassen überlegen,

Trauma Berufskrankh 2017 · 19 (Suppl 3):S250-S254 DOI 10.1007/s10039-017-0287-1

(c) Springer Medizin Verlag GmbH 2017

\section{J. Zeichen \\ Notwendige Weiterentwicklung aus der Sicht des D-Arztes am Krankenhaus}

\section{Zusammenfassung}

Mit der Neuausrichtung des stationären Heilverfahrens 2014 erfolgte eine Neuordnung der Patientenversorgung. Unter anderem sollte dadurch die Qualität der Behandlung verbessert werden. Die Unfallversicherungsträger haben mit der Neuausrichtung mehr Einfluss in der Behandlung von Patienten bekommen. Es ist zu einer Zunahme der Bürokratie gekommen. Inwieweit es zu Verbesserungen der Qualität gekommen ist, ist bisher nicht bekannt. Zur Weiterentwicklung der derzeitigen Situation sind Veränderungen der Rahmenbedingungen notwendig. Der bürokratische Aufwand muss verbessert werden. Das Datenübermittlungsverfahren (DALE-UV) sollte überarbeitet werden mit intelligenten
IT-Lösungen. Eine intensive Diskussion der Qualitätsmessung ist erforderlich. Lösungen müssen erarbeitet werden, damit v. a. komplexe Fälle ausreichend vergütet werden. Die Aus- und Weiterbildung in den chirurgischen Disziplinen hat sich in den letzten Jahren verändert. Es müssen Strukturen geschaffen werden, damit die Aus- und Weiterbildung im D-Arzt-Verfahren adäquat für die Zukunft vorbereitet ist. Zur Weiterentwicklung des D-Arztes im Krankenhaus ist also eine Vielzahl von Veränderungen und Verbesserungen notwendig.

Schlüsselwörter

Bürokratie · Qualität · Vergütung · Ausbildung · Weiterbildung

\section{Necessary further development from the perspective of accident insurance consultants in hospitals}

\section{Abstract}

With the reorientation of the German statutory accident insurance for inpatient treatment in 2014, the patient care was reorganized. Among other things, the quality of the treatment should be improved. With the new orientation, the accident insurance providers have gained more influence in the treatment of patients. There has been an increase in bureaucracy. The extent to which improvements in quality have occurred is not yet known. In order to further improve the current situation, changes in the basic conditions are necessary. The bureaucratic effort has to be improved. The data transmission process (DALE-UV) should be revised with intelligent information technology (IT) solutions. An intensive discussion on the quality measurement is necessary. Solutions must be developed to ensure that complex cases are adequately compensated. Training in the surgical disciplines has changed in recent years. Structures must be created to ensure that the training and further education in the accident insurance consultant procedure are adequately prepared for the future; therefore, a large number of changes and improvements are necessary to develop the accident insurance consultant procedure in hospitals.

Keywords

Bureaucracy · Quality · Compensation .

Education · Training den Katalog des Verletzungsartenverfahrens zu übernehmen. Dann wird sich die Behandlung vieler Patienten nur mehr in den großen Häusern konzentrieren. Für die Außenwirkung der größeren Kliniken ist dies gut. Was passiert aber mit kleineren Kliniken? Es wird natürlich zu einer weiteren Reduktion kleinerer Kliniken kommen. Ob dies so politisch gewollt ist, bleibt abzuwarten.
Um Qualität ggf. messen zu können, sollten Register aufgebaut werden, wie dies in der DGOU (Deutsche Gesellschaft für Orthopädie und Unfallchirurgie) der Fall ist [11]. Dabei gibt es eine indirekte Evidenz, dass dadurch die Ergebnisund Versorgungsqualität verbessert wird [14]. Bisher gibt es keine Daten, dass es durch die Veränderung des Heilverfahrens zu einer Verbesserung der Ergebnisqualität gekommen ist. Es ist deshalb 


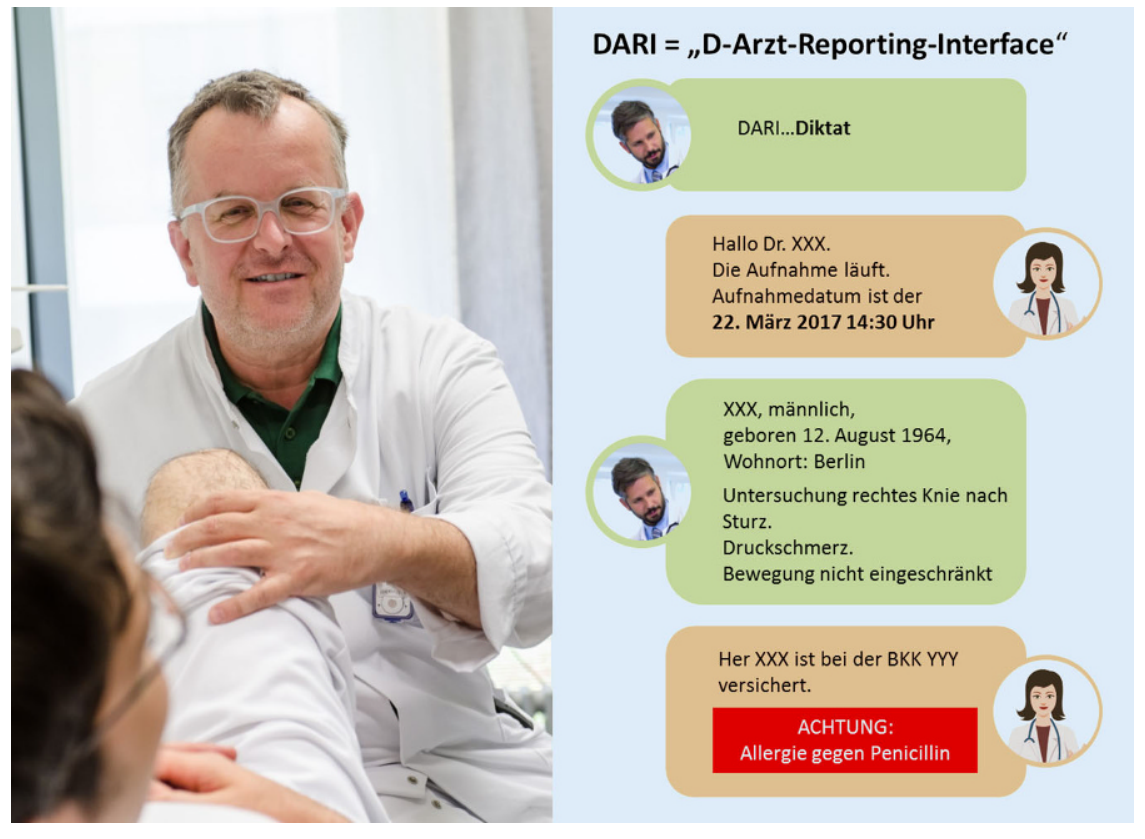

Abb. 2 \ D-Arzt-Reporting-Interface (DARI)

auch der Aufbau von Datenbanken zu fordern. Dadurch erfolgt eine Transparenz in der Behandlung.

Bei der Behandlung berufsgenossenschaftlicher Patienten haben heutzutage immer mehr die Versicherungsträger das Sagen. Es erfolgen eine strengere Überwachung und Kontrolle des Heilverfahrens. Meines Erachtens besteht dadurch kein Benefit in der Behandlung. Mittlerweile wird auch die Qualität der Durchgangsarztberichte geprüft. Dabei wurde für ein Peer-Review-Verfahren eine Checkliste zur Begutachtung von Durchgangsarztberichten entwickelt. Bei der Erprobung der Checkliste unter Realbedingungen betrug die Berichtsnote im Mittel 2,6 $+1,1(1=$ sehr gut, $5=$ ungenügend) für insgesamt 474 begutachtete Durchgangsarztberichte. Am häufigsten bemängelt wurde die Schlüssigkeit der Art der eingeleiteten Heilbehandlung [15]. Derzeit soll die Qualität der Durchgangsarztberichte in einer größeren Anzahl geprüft werden. Sicherlich ist dies ein guter Ansatz zur Verbesserung der Dokumentationsqualität. Was passiert mit den Kollegen, die eine schlechte Dokumentationsqualität abliefern? Es ist $\mathrm{zu}$ vermuten, dass zunächst Gespräche geführt werden mit Sanktionen, wenn keine Verbesserung im weiteren Verlauf eintritt. Damit können die Versiche- rungsträger noch mehr Druck ausüben. Die Ergebnisqualität wird dadurch aber nicht verbessert. Allein durch die derzeit regelmäßig erforderlichen Fortbildungen wird sich meines Erachtens auch nicht die Dokumentationsqualität in der Zukunft verbessern. Hier bedarf es auch intelligenter IT-Lösungen.

Durch verschiedene Umstände sind die Zahlen der Arbeitsunfälle in den letzten Jahren zurückgegangen. Entgegengesetzt dazu sind die Aufwendungen für Entschädigungsleistungen kontinuierlich angestiegen [6]. Woran kann das liegen? Die Anspruchshaltung der Patienten ist gestiegen. Jeder kennt aus eigener Erfahrung Patienten, die ein re Entschädigungen erhalten. Hier soll und muss eine Verbesserung erfolgen. Die Unfallversicherungsträger sind kein Wohlfahrts- oder Wohlfühlunternehmen. Das Reha-Management stellt dazu eine gute Entwicklung dar. Hier sollte überprüft werden, ob es dadurch zu einer Reduktion der Entschädigungsleistungen kommt.

Das Thema Qualität stellt sicherlich einen der schwierigsten Bereiche dar. Allein Kennzahlen festzulegen und daran Leistungen kontinuierlich zu optimieren ist meiner Meinung nach zu wenig, um die Qualität zu verbessern. Ob z. B. ein "Arzt-Hopping" machen, damit sie ih-
Qualitätssiegel hierzu ein Mittel darstellt, bleibt fraglich. Man erfüllt dabei als Leistungsanbieter eine Liste von definierten Kriterien, ohne dass es den meisten Nutzern klar ist, wofür das Siegel eigentlich genau steht und welchen inhaltlichen Wert es wirklich hat. Es bedarf weiterer und intensiver Diskussionen um die Qualität der Patientenversorgung. Durch die enorme Bürokratie mit Reduktion von Personal und Zeit fehlt immer mehr die interaktive Kommunikation. Notwendig wäre deshalb eine Verbesserung der Kommunikationsqualität. Meines Erachtens ist dies ein Bereich, mit dem man auch die Behandlung von Patienten verbessern kann.

\section{Vergütung}

Die Behandlung komplexer Fälle wird im heutigen DRG-System nicht ausreichend abgebildet. Bonnaire et al. [3] haben in ihrer Publikation festgestellt, dass der Aufwand an struktureller und personeller Vorhaltung im SAV-Verfahren gestiegen ist, ohne dass es zu einer Erlösverbesserung für die Krankenhäuser gekommen ist. Bei Patienten mit notwendigen mehrfachen operativen Versorgungen belastet dies die Kliniken. Es ist anzuregen, dass wie bei anderen Versorgungsfällen ein Zusatzentgelt eingeführt wird, damit die Behandlung kostendeckend erfolgen kann.

\section{Ausbildung}

Ein generelles, vielschichtiges Problem stellen die Aus- und Weiterbildung dar. Mit der Reform 2011 wurden 3 Stufen der D-Arzt-Qualifikation eingeführt (•Tab. 1). Durch die Veränderung im Heilverfahren wird es für Auszubildende immer schwieriger, einen geeigneten Ausbildungsplatz $\mathrm{zu}$ bekommen. Wie Kalbe [9] festgestellt hat, stellt besonders der Nachweis einer 1-jährigen Tätigkeit in einem VAV-Haus im Anschluss an die Facharztprüfung eine Hürde dar, wenn die Kollegen eine Praxis übernehmen wollen. Absehbar ist es, dass es dadurch im ländlichen Bereich zu einer Reduktion von D-Arzt-Praxen kommt.

Um dem entgegenzuwirken, ist meines Erachtens zunächst eine Bedarfsana- 


\section{Übersicht}

\section{Tab. 1 Systematik der D-Arzt-Beteiligung seit 2011}

\section{Bezeichnung}

D-Arzt Weiterbildung

Facharzt für Orthopädie und Unfallchirurgie plus mindestens $1 \mathrm{Jahr}$ VAV-Tätigkeit danach

D-Arzt mit operativer Tätigkeit

Wie oben mit Zusatzweiterbildung spezielle Unfallchirurgie

D-Arzt VAV- oder SAV-Klinik

Wie oben, zusätzlich mindestens 3 Jahre Tätigkeit

\section{Operationsspektrum}

Auf bestimmte ambulante Operationen begrenzt

Alle Operationen außerhalb des SAV

Alle operativen Eingriffe, für welche die Klinik zugelassen ist

VAV Verletzungsartenverfahren, SAV Schwerverletztenartenverfahren

lyse notwendig. Auch in diesem Bereich müssen intensive gemeinsame Diskussionen erfolgen, damit ausreichend gut ausgebildete D-Ärzte in der Zukunft vorhanden sind. In der heutigen Zeit ist es auch immer schwieriger, Mitarbeiter zu gewinnen. Vielleicht hilft hier auch eine finanzielle Unterstützung der Unfallversicherungsträger, damit die Qualität in der Aus- und Weiterbildung erhalten werden kann. Da es bisher in Deutschland überhaupt keine Vergütung bei der Aus- und Weiterbildung gibt, könnten hier vielleicht die Unfallversicherungsträger eine Vorreiterrolle übernehmen. Auch sollten die erforderlichen Ausbildungskataloge regelmäßig überprüft und angepasst werden.

\section{Fazit für die Praxis}

- Viele Veränderungen haben im stationären Heilverfahren in den letzten Jahren stattgefunden. Es erfolgte eine Ordnung im System mit Zunahme der Spezialisierung und Konzentration der Patientenversorgung. Struktur- und Prozessanforderungen wurden geschaffen.

- Der Bürokratieaufwand ist unter Zuhilfenahme intelligenter IT-Lösungen zu verbessern. Der Ist-Zustand muss kritisch überprüft werden.

- Eine intensive Diskussion der Qualität in Bereichen der Patientenversorgung ist notwendig. Eine Verbesserung der Kommunikationsqualität wäre wünschenswert.

- Die Vergütungsstruktur muss der Realität angepasst werden.

- Bei der Aus- und Weiterbildung zukünftiger D-Ärzte könnten die Unfallversicherungsträger eine Vorreiterrolle übernehmen, wenn hier finanzielle Ressourcen geschaffen werden.

- Nur durch ständige Analysen und daraus folgende gemeinsame Lösungsstrategien der Ärzte mit den Unfallversicherungsträgern können ggf. Strukturen geschaffen werden, die zu einer flächendeckenden Verbesserung der Patientenversorgung führen werden.

\section{Korrespondenzadresse}

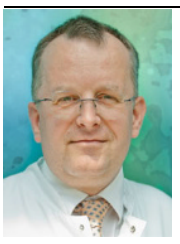

Prof. Dr. J. Zeichen

Johannes Wesling Klinikum

Minden

Hans Nolte Str. 1

32429 Minden, Deutschland Johannes.Zeichen@ muehlenkreiskliniken.de

\section{Einhaltung ethischer Richtlinien}

Interessenkonflikt. J. Zeichen gibt an, dass kein Interessenkonflikt besteht.

Dieser Beitrag beinhaltet keine vom Autor durchgeführten Studien an Menschen oder Tieren.

The supplement containing this article is not sponsored by industry.

\section{Literatur}

1. Andro KH (2002) DALE-UV Projekt zur Datenübermittlung. Trauma Berufskrankh 4(Suppl 3):219-222

2. Baumann F, Mahr D, Neumann C, Nerlich M (2016) Neuordnung des stationären Heilverfahrens der Gesetzlichen Unfallversicherung: Aus Sicht einer Universitätsklinik. Unfallchirurg 119:901-907

3. Bonnaire F, Goepel M, Bula P (2016) Die neuen Heilverfahren der DGUV: Aus der Sicht eines Städtischen Krankenhauses. Unfallchirurg 119:908-914

4. Bühren V, Perl M (2016) Die neuen Heilverfahren der DGUV: Aus Sicht der BG Kliniken. Unfallchirurg 119:895-900
5. Bundesministerium für Gesundheit (2016) Entbürokratisierung in der Pflegedokumentation. https://www.bundesgesundheitsministerium.de/ themen/pflege/entbuerokratisierung.html.Zugegriffen: 18. Juni 2017

6. Deutsche Gesetzliche Unfallversicherung (2015) Veröffentlichungen. http://www.dguv.de/de/ zahlen-fakten/veroeffentlichungen/index.jsp. Zugegriffen:25. Juni 2017

7. Deutsche Gesetzliche Unfallversicherung (2016) Datenaustausch mit Leistungserbringern in der gesetzlichen Unfallversicherung. http://www. dguv.de/medien/dale-uv/documents/download/ aerzte/flyer_2016-05-13.pdf.Zugegriffen:25.Juni 2017

8. Hartmannbund (2017) Umfrage wirft beunruhigende Schlaglichter auf die Arbeitssituation von Assistenzärzte. http://www.hartmannbund.de/ detailansichten/pressemitteilungen/meldung/ umfrage-wirft-beunruhigende-schlaglichterauf-die-arbeitssituation-von-assistenzaerzten/. Zugegriffen: 18. Juni 2017

9. Kalbe P (2016) Neuordnung des BG-lichen Heilverfahrens aus der Sicht eines niedergelassenen D-Arztes. Unfallchirurg 119:915-920

10. Kleinschmidt F (2007) Chirurgische Organisationsformen und berufsgenossenschaftliches Regelwerk. Trauma Berufskrankh 9(Suppl3):S333-S225

11. Kostuj T, Kladny B, Hoffmann R (2016) Die Register derDGOU.Unfallchirurg 119:463-468

12. Mand C, Müller T, Ruchholtz S et al (2012) Organisatorische, personelle und strukturelle Veränderungen durch die Teilnahme am TraumaNetzwerkD DGU. Unfallchirurg 115(5):417-426. doi:10.1007/s00113-010-1886-5

13. Sarkar MR (2016) Erfahrungen einer Durchgangsarztverfahren (DAV) Klinik. Trauma Berufskrankh 18(Suppl4):S297-S300

14. Stengel D, Dreinhöfer K, Kostuj T (2016) Einfluss von Registern auf die Versorgungsqualität. Unfallchirurg 119:482-487

15. Szczotkowski D, Brodowski H, Nolting Hetal (2017) Entwicklung und Erprobung einer Checkliste zur Beurteilung der Qualität von Durchgangsarztberichten. Trauma Berufskrankh 19:41-46 